\title{
Correlación de calcio total, calcio iónico como valor pronóstico de hipoparatiroidismo posterior a tiroidec- tomía total en el Instituto de Previsión Social Hospital Central- Paraguay - 2019
}

\author{
Correlation of total calcium, ionic calcium as a prognostic value of \\ hypoparathyroidism after total thyroidectomy at the \\ Instituto de Previsión Social Hospital Central - Paraguay - 2019
}

Osvaldo Gregorio Quintana González ${ }^{1}$; Roque Rafael Rodríguez Leiva ${ }^{1}$; Jessica Gill Oliveira ${ }^{1}$; Angélica María Jatchuk Luchuk ${ }^{1}$; Mauricio Nicolás Barreto Ríos ${ }^{1}$; Joel Iván López Mendoza ${ }^{1}$

\section{RESUMEN}

Introducción: La hipocalcemia es una complicación frecuente posterior a tiroidectomía total, el dosaje de la paratohormona en el posoperatorio resulta importante para identificar el riesgo de hipocalcemia sintomática precoz pos-tiroidectomía total, empero es importante identificar otros tipos de indicadores. Objetivo: correlacionar el dosaje de calcio total y calcio iónico con el dosaje de paratohormona como valor pronóstico de hipoparatiroidismo en pacientes sometidos a tiroidectomía total en el servicio de Cirugía General del Hospital Central del Instituto de Previsión Social en el periodo de junio 2018 a junio 2019. Metodología: Estudio descriptivo retrospectivo, en el que se evaluaron factores como datos demográficos, clínicos, laboratoriales y anatomopatológicos. Se emplearon las fichas médicas, quirúrgicas y de consultorio. Resultados evaluaron a 58 pacientes, 93,1\%: Se eran mujeres. El promedio de edad fue de $52 \pm 14$ años. El 65,5\% de los pacientes presentaron normocalcemia, el 13,8\% Hipoparatiroidismo transitorio (HT) y el 20,7\% Hipoparatiroidismo permanente (HP). Previa a la operación los pacientes presentan dosaje normal de calcio total (CAT) e ionizado (CAI); cabe resaltar que los pacientes con HP presentan dosaje de CAT y CAI menores en todos los controles y los pacientes con HT muestran un aumento al tercer control (96hs). Conclusión: Un paciente con hipocalcemia determinado con CAI a las 24h mostró un factor de riesgo de 90 veces más de HP. La medición de CAT 96hs y de CAI 24hs, mostraron una correlación alta de predecir el HP medido por PTH ( $r=0,734$ y r=0,719, respectivamente).

Palabras clave:Hipoparatiroidismo, Tiroidectomía, Trastornos del Metabolismo del Calcio.

Fecha de recepción: agosto 2020; fecha de aceptación: octubre 2020

${ }^{1}$ Instituto de Previsión Social, Hospital Central. Asunción, Paraguay

Autor de correspondencia: María Jatchuk Luchuk. Email: jatchukangelica@gmail.com 


\section{ABSTRACT}

Introduction: Hypocalcaemia is a frequent complication after total thyroidectomy, postoperative dosing of parahormone is important to identify the risk of early symptomatic hypocalcaemia after total thyroidectomy, but it is important to identify other types of indicators. Objective: to correlate the dose of total calcium and ionic calcium with the dose of paratohormone as a prognostic value of hypoparathyroidism in patients undergoing total thyroidectomy in the General Surgery service of the Central Hospital of the Social Welfare Institute from June 2018 to June 2019. Methodology: Retrospective descriptive study, in which factors such as demographic, clinical, laboratory and pathological data were evaluated. Medical, surgical and office records were used. Results: 58 patients were evaluated, 93 were women. The average age was $52 \pm 14$ years. $65.5 \%$ of the patients presented normocalcaemia, 13.8\% Transient hypoparathyroidism (HT) and $20.7 \%$ Permanent hypoparathyroidism (HP). Before the operation, the patients present normal total calcium (CAT) and ionized calcium (CAI); It should be noted that patients with HP present lower CAT and CAI dosages in all controls and patients with HT show an increase in the third control (96hs). Conclusion: A patient with hypocalcaemia determined with IAC at $24 \mathrm{~h}$ showed a risk factor of 90 times more than HP. The measurement of CAT 96hs and CAI 24hs, showed a high correlation to predict the HP measured by PTH $(r=0.734$ and $r=0.719$, respectively $)$.

Keywords: Hypoparathyroidism, Thyroidectomy, Calcium Metabolism Disorders 


\section{INTRODUCCIÓN}

La glándula paratiroidea son cuatro estructuras ubicadas posteriormente de la glándula tiroides; cuya función endocrina es la secreción de la hormona paratiroidea (PTH) indispensable para función primaria la regulación homeostática del calcio. La hormona actúa directamente sobre el hueso produciendo la liberación de calcio; en los riñones, intensifica la reabsorción de dicho ion en el túbulo distal y proximal donde sintetiza 1,25-dihidroxivitamina D $(1,25[\mathrm{OH}] 2 \mathrm{D})$, hormona que intensifica la absorción de calcio por vía gastrointestinal $(1,2)$.

El hipoparatiroidismo frecuentemente se debe a la extirpación quirúrgica involuntaria de las glándulas paratiroideas, correspondiendo al $75 \%$ de todos los casos de hipoparatiroidismo. Las cirugías asociadas a este trastorno incluyen la tiroidectomía total y cirugía radical de cuello por neoplasias malignas de cabeza y cuello. La paratiroidectomía, por uno o más nódulos paratiroideos, podrían resultar en hipoparatiroidismo. Se mencionan otras causas de hipoparatiroidismo mucho menos frecuentes tales como destrucción autoinmunitaria de la tiroides (suele se asociada con un síndrome poliglandular), genéticas, infiltración de la paratiroides, tratamiento ablativo con radioyodo, síndrome del hueso hambriento e infección por VIH y sepsis por gramnegativos. Se ha observado que en algunos casos no se ha eliminado todo el tejido, pero el que queda sufre un deterioro del riego sanguíneo que resulta finalmente en hipoparatiroidismo $(3,4)$.

La presencia de hipocalcemia indica una falla de la acción homeostática de la PTH, dando lugar a la aparición de manifestaciones neuromusculares y neurológicas que consisten en espasmos musculares, espasmos carpopedales gesticulación facial y en casos extremos, espasmos laríngeos y con- vulsiones. Puede haber paro respiratorio. En algunos pacientes sobreviene hipertensión intracraneal asociada a edema de papila y alteraciones mentales como irritabilidad, depresión y psicosis. El intervalo QT del electrocardiograma esta prolongado e inversión de la onda T. Para confirmar la tetania latente se pueden explorar los signos de Chvostek o de Trousseau. El signo de Chvostek es poco sensible no específico para hipocalcemia, aproximadamente 10,0 a $25,0 \%$ de los individuos sanos tendrán positivo este signo y pudiendo ser negativo en aproximadamente $30 \%$ de los pacientes con hipocalcemia. En contraste, el signo de trousseau tiene una sensibilidad aproximada del 90,0 a 99,0\% y está presente solo en el 1,0\% de los pacientes hipocalcémi$\cos (5,6)$.

Las incidencias de hipocalcemia y de hipoparatiroidismo pueden estar influenciadas por diferentes factores, lo cual producen una mayor estancia hospitalaria, y por ende, costos más elevados en la atención de la salud (7-9).

La reducción calcio sérico durante las primeras 24 horas posoperatorio predice de manera fiable, económica y rápida el desarrollo de hipoparatiroidismo definitivo, mientras que niveles bajos de calcio ionizado.

ya sea inmediato o largo plazo como factor predictor es mucho más específicol, sin embargo, el descenso de la PTH durante las primeras 24 horas posoperatorio o intraoperatorio es un determinante aún más específico como factor pronóstico (10-12).

Teniendo en cuenta alto porcentaje de hipoparatiroidismo como complicación frecuente en pacientes sometidos a tiroidectomía total y teniendo en cuenta la necesidad de lograr el alta medico con mayor optimización, es importante identificar aquellos pacientes con mayor riesgo a hipocalcemia, mediante técnicas que puedan ser más eficaces y eficientes para el Hospital y 
que permitan lograr un protocolo eficaz de tratamiento para el paciente (13).

Objetivo General: Correlacionar el dosaje de calcio total y calcio iónico con el dosaje de paratohormona como valor pronóstico de hipoparatiroidismo en pacientes sometidos a tiroidectomía total en el servicio de Cirugía General del Hospital Central Dr. Emilio Cubas del Instituto de Previsión Social en el periodo de junio 2018 a junio 2019. Objetivos Específicos: caracterizar a los pacientes según datos demográficos, datos clínicos y tiempo de internación en los pacientes posoperados; valorar el estudio citológico y anatomopatológico tiroideo; evaluar la funcionali-

\section{MÉTODO}

Fue un estudio analítico longitudinal retrospectivo con datos secundarios con una población enfocada de pacientes atendidos en el Instituto de Previsión Social Hospital Central posoperados de tiroidectomía total durante el periodo de junio del 2018 a junio del 2019 con seguimiento por consultorio ambulatorio de clínica médica.

\section{Criterios de Inclusión}

Pacientes internados con indicación de tiroidectomía total en el Servicio de Cirugía General, por diferentes motivos. Pacientes con ficha de evaluación pre quirúrgica completa. Pacientes con seguimiento clínico posterior a la cirugía en consultorio ambulatorio de clínica médica.

\section{Criterios de Exclusión}

Pacientes con insuficiencia renal crónica en cualquier estadio, con albumina sérica por debajo del valor normal teniendo en cuenta que estos parámetros podrían llevar a sesgos en la interpretación de los resultados. Pacientes con seguimiento irregular posterior a cirugía, es decir que no cumplan con las citas previamente fijadas. dad tiroidea previa a la cirugía; registrar el dosaje de calcio sérico, ionizado, fosforo, magnesio y hormona paratiroidea en el periodo pre y posoperatorio; establecer porcentaje de hipoparatiroidismo transitorio y permanente; comparar el dosaje de calcio sérico e ionizado según normocalcemia, hipoparatiroidismo transitorio y permanente; identificar los signos y síntomas de hipocalcemia posoperatorio en pacientes con Hipoparatiroidismo; evaluar la razón de probabilidades de la hipocalcemia antes de los 6 meses con respecto al Hipoparatiroidismo permanente; y correlacionar el dosaje de calcio total e iónico con la hormona paratiroidea.

El muestreo fue no probabilístico de casos consecutivos. Se identificaron los pacientes que fueron sometidos a tiroidectomia total en el servicio de Cirugía General, de entre los mismos, se seleccionarán aquellos que reúnan con los criterios de selección, luego del postoperativo, se verificó que las fichas de los pacientes presentaran los datos bioquímicos requeridos en el tiempo considera máximo, es decir $24 \mathrm{~h}$ posteriores a la cirugía. Luego de la alta médica, se verificó el cumplimiento de los controles ambulatorios con controles laboratoriales solicitados y ficha clínica hasta el sexto mes posoperatorio. Se diseñó un registro electrónico para la recopilación de datos de acuerdo a los datos requeridos para el cumplimiento de los objetivos. El mismo contó con los datos: demográficos, clínicos, bioquímicos, quirúrgicos y otros. La fuente de información fueron los expedientes físicos y digitales de pacientes, que incluyan aspectos clínicos, laboratoriales y técnicas quirúrgicas y ficha de consultorio. 


\section{RESULTADOS}

Se revisaron 58 fichas de pacientes, de los cuales el 93,1\% eran del sexo femenino. El promedio de edad de la muestra fue de $52 \pm 14$ años, siendo los hombres en promedio mayores que las mujeres, $65 \pm 11$ años y $51 \pm 14$ años, respectivamente. El 77,6\% de los pacientes refirieron residir en el área urbana En cuanto a los datos clínicos, el $63,8 \%$ de los pacientes eran hipertensos, según su ficha médica. La mediana de

Tabla 1: Función tiroidea previa a la cirugía.

\begin{tabular}{|crr|}
\hline $\begin{array}{l}\text { Parámetros bioquími- } \\
\text { cos relacionadas a la } \\
\text { función tiroidea }\end{array}$ & $\mathbf{X} \pm \mathbf{D E}$ & Me-RIC \\
\hline $\mathrm{TSH}(\mathrm{mIU} / \mathrm{L})$ & $1,9 \pm 1,9$ & $1,7-2,9$ \\
$\mathrm{~T} 4(\mathrm{pg} / \mathrm{mL})$ & $13,3 \pm 3,6$ & $12,3-4,7$ \\
$\mathrm{~T} 3(\mathrm{mmol} / \mathrm{L})$ & $2,1 \pm 2$ & $1,8-0,5$ \\
\hline
\end{tabular}

Según los registro médicos, el 67,2\% de los pacientes sometidos a cirugía fueron clasificados como "Benignos" según su composición, la estructura y las caracterís-

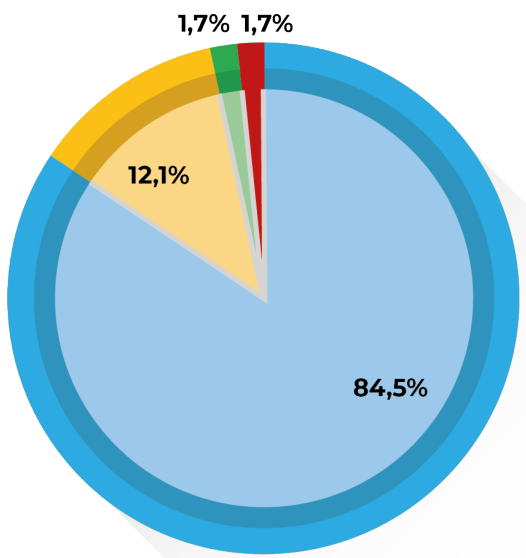

Gráfico 2: Clasificación según Ecografía tiempo de internación fue de 3 días.

En la tabla 1 se puede observar los datos cuantitativos y cualitativos de la funcionalidad tiroidea, según el promedio de TSH, T4 y T3, los pacientes presentan función normal. Al clasificar, evaluar la función de la hormona previa a la cirugía fue de un $74,1 \%$ "Normofuncionante" y solo 1 paciente presentó hipotiroidismo $(1,7 \%)$. (Gráfico 1)

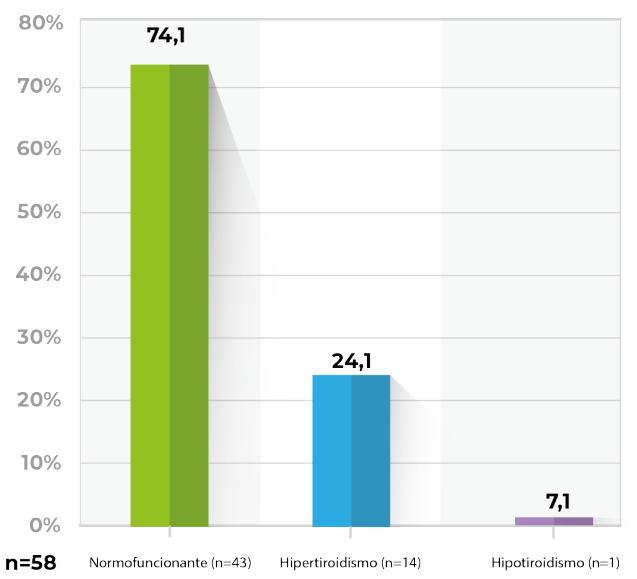

Gráfico 1: Clasificación de la función de la tiroides

ticas del tejido. Según Anatomía Patológica el 53,4\% presentan Bocio Coloide Multinodular y según ecografía, el 84,5\% de presentó "Bocio difuso" (Gráficos 2 y 3)

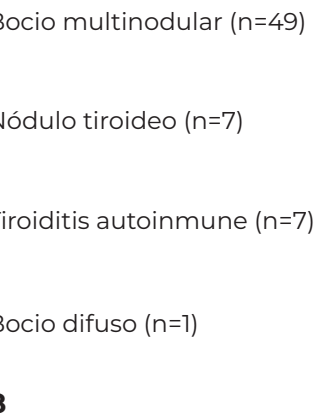




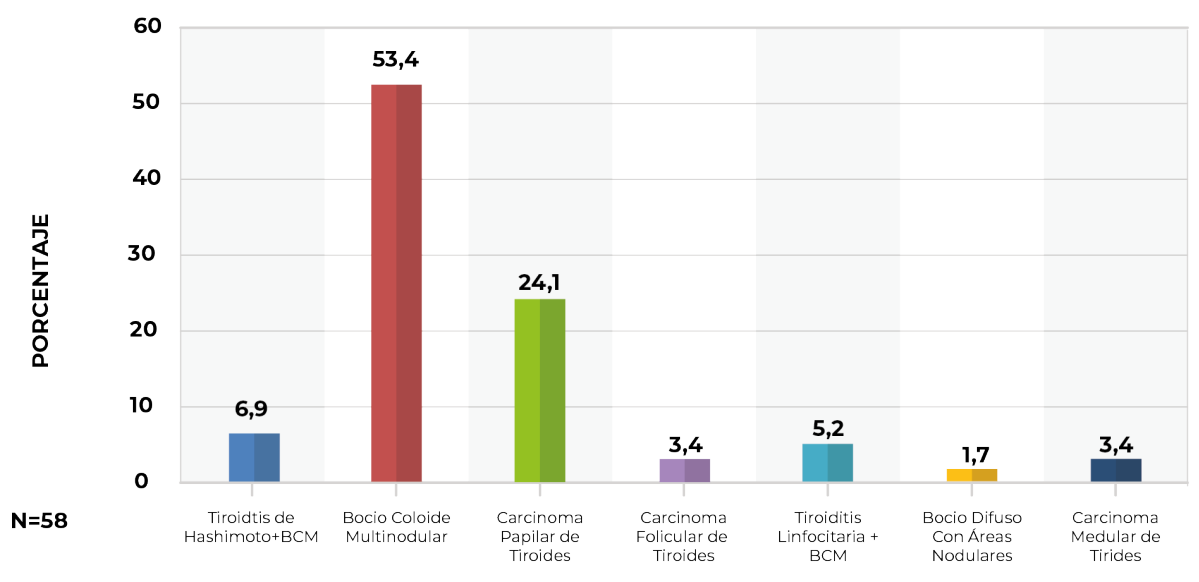

Gráfico 3: Clasificación según anatomía patológica

En cuanto al dosaje de calcio, se pue- bien antes de la cirugía $(54,3 \pm 7,8 \mathrm{pg} /$ de observar un descenso en el prome- $\mathrm{mL})$ y a los 6 meses $(44,9 \pm 21,8 \mathrm{pg} /$ dio en los valores de Calcio total e ióni- $\mathrm{mL}$ ) presentaron valores dentro del ranco a las $24 \mathrm{hs}$ y vuelve a aumentar desde go, se puedo observar que el promeel control 96hs. En cuanto a la PHT si dio fue menor a los 6meses. (Gráfico 4).

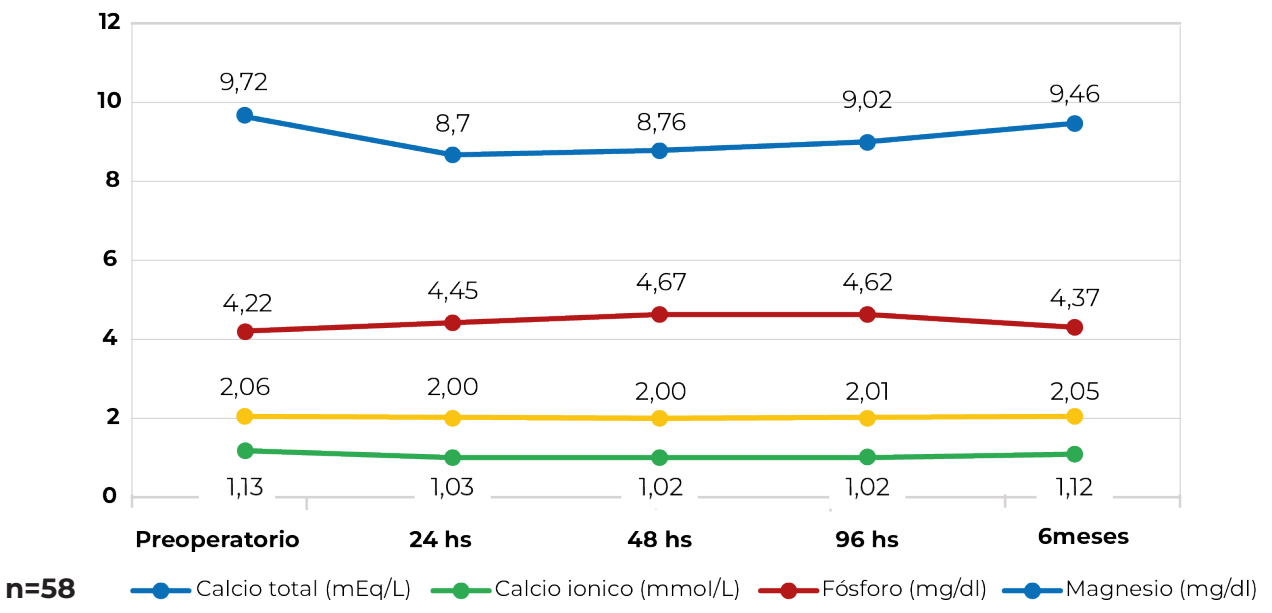

Gráfico 4: Dosaje de calcio sérico, ionizado, fosforo, magnesio y hormona paratiroidea en el periodo pre y posoperatorio

El $65,5 \%$ de los pacientes presentaron Normoparatiroidismo. En cuanto al hipoparatiroidismo, resultó llamativo que el porcentaje de pacientes con permanente fue más que el transitorio, $20,7 \%$ y $13,8 \%$ respectivamente.

En el Gráfico 5, se puede ver la evolución del dosaje de calcio total (CAT) en función al diagnóstico final a los 6 meses de hipoparatiroidismo, se puede observar que todos los pacientes presentan valores similares de CAT previa a la cirugía ( $\mathrm{p}>0,05)$, se observa un descenso a las $24 \mathrm{hs}$, siendo el pico menor a las $48 \mathrm{hs}$, cabe resaltar que promedio ascendente desde el control de CAT a las 96hs. En todos los controles el 
promedio de CAT es menor, siendo estos valores significativamente en los controles postoperatorios inmediatos $(24 \mathrm{hs}, 48 \mathrm{hs} \mathrm{y}$ 96hs); mientras que al comparar entre los HT y HP las diferencias no son significa- tivas en los controles realizados a las $24 \mathrm{hs}$ y $48 \mathrm{hs}(\mathrm{p}>0,05)$, es decir los promedios se comportan igual y el aumento observado en los pacientes HT son significativamente mayores a las $96 \mathrm{hs}(\mathrm{p}<0,05)$.

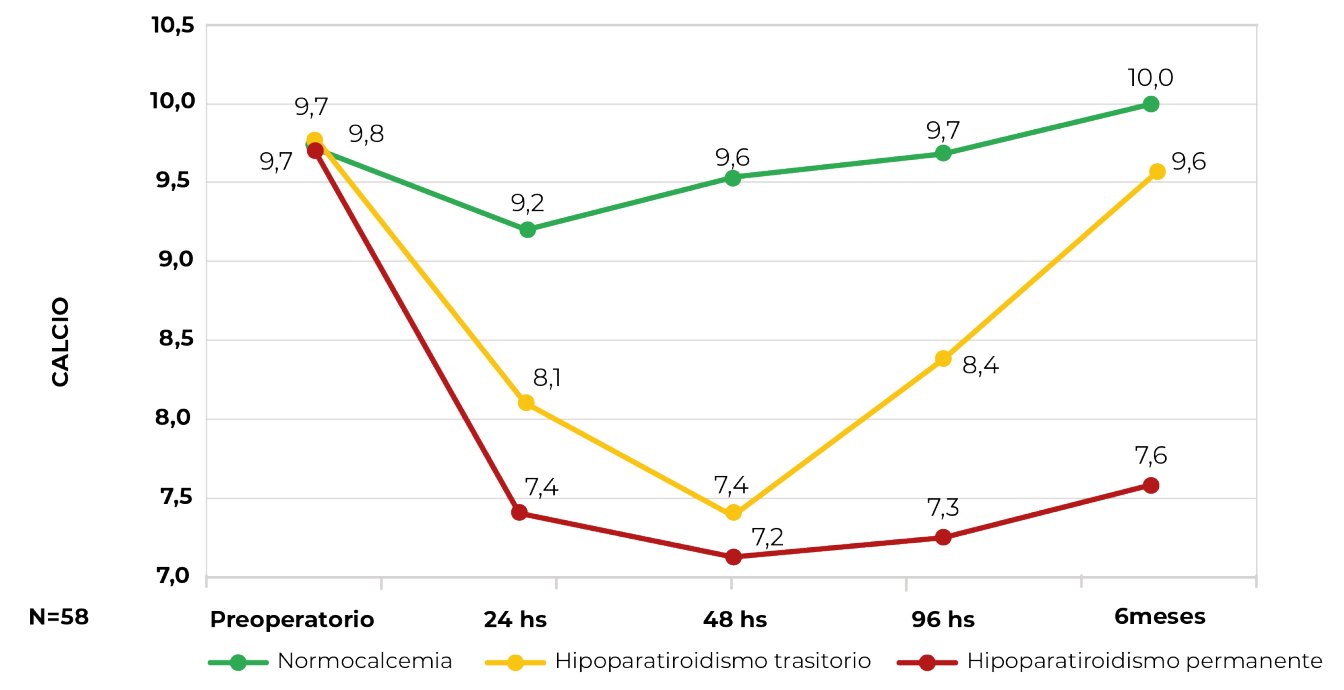

Gráfico 5: Evolución de calcio total según normocalcemia, hipoparatiroidismo transitorio (HT) $y$ permanente $(H P)$.

En la evolución del dosaje de calcio iónico (CAI), se puede observar que todos los pacientes presentan valores similares de CAI previa a la cirugía ( $p>0,05)$, en los pacientes normocalcemicos presentaron promedios iguales a las $24 \mathrm{hs}, 48 \mathrm{hs}$ y $96 \mathrm{hs}$, alcanzó su pico mayor a las 6meses. Mientras que en los pacientes hipoparatiroideos, se observa un descenso a las $24 \mathrm{hs}$, siendo el pico menor a las $48 \mathrm{hs}$, cabe resaltar que promedio de CAI ascendente desde el control las 96hs. En todos los controles el promedio de CAI es significativamente menor $(\mathrm{p}>0,05)$ en los controles postoperatorios inmediatos $(24 \mathrm{hs}$, $48 \mathrm{hs}$ y $96 \mathrm{hs}$ al comparar el dosaje entre los HT y HP las diferencias no son significativas en los controles realizados a las $24 \mathrm{hs}$ y $48 \mathrm{hs}(\mathrm{p}>0,05)$ entre ellos, pero si entre los pacientes normocalcemicos $(p<0,05)$. A los 6 meses los promedios siguen manteniéndose significativamente diferentes $(\mathrm{p}<0,05)$ (Gráfico 6).

Se pudo observar que el $22,4 \%$ de los pacientes presentó Parestesia y 20,7\% presentó Trosseau y Chvostek. En cuanto a los síntomas, la totalidad de los pacientes con HP presentaron signos de Chvostek, Trosseau y Parestesia; mientras que el $75 \%$ presentó Chvostek y Parestesia y un $62,5 \%$ Trosseau de los pacientes con HT y Solo 1 paciente presentó signos el cual correspondió a Parestesia (Gráfico 7). 


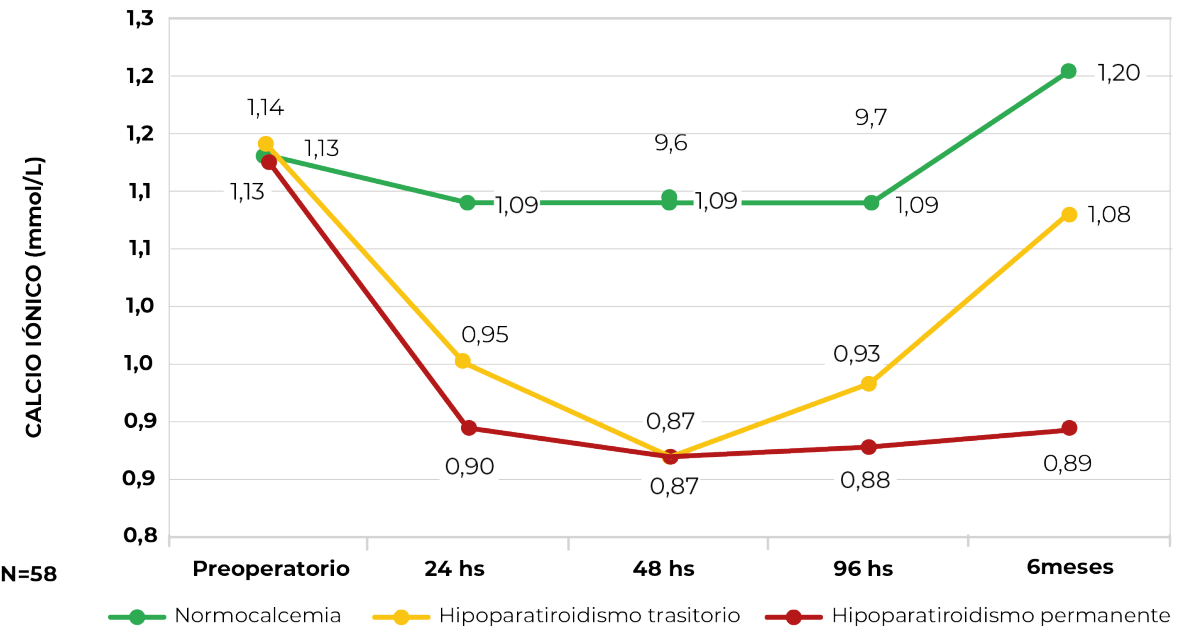

Gráfico 6: Evolución de calcio total según normocalcemia, hipoparatiroidismo transitorio (HT) y permanente $(H P)$.

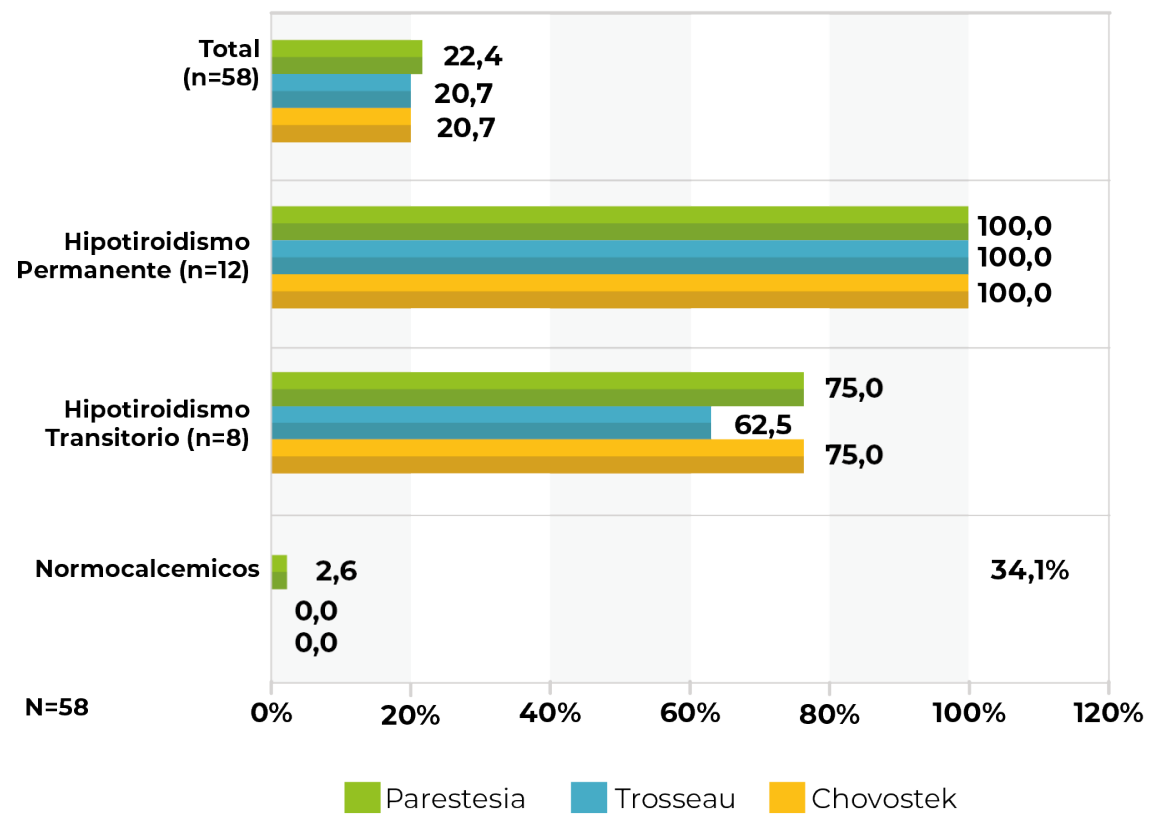

Gráfico 7: Signos y síntomas de hipocalcemia según normocalcemia, hipoparatiroidismo transitorio (HT) y permanente (HP) a los 6 meses.

Se observó que todos los pacientes con HP y HT son todas mujeres, los pacientes normocalcémicos, corresponden a $89,5 \%$ de mujeres, todos los hombres que participaron de la investigación fueron normocalcémicos (Gráfico 8).

Los pacientes con $\mathrm{H}$, el $66,7 \%$ presentó según análisis histológico de "Benigno", mientras que los pacientes con HT la mitad corresponden a resultados "Malignos" según Histología y el 71,1\% de los pacientes NC fueron diagnosticados según histología como “Benignos” (Gráfico 9). 


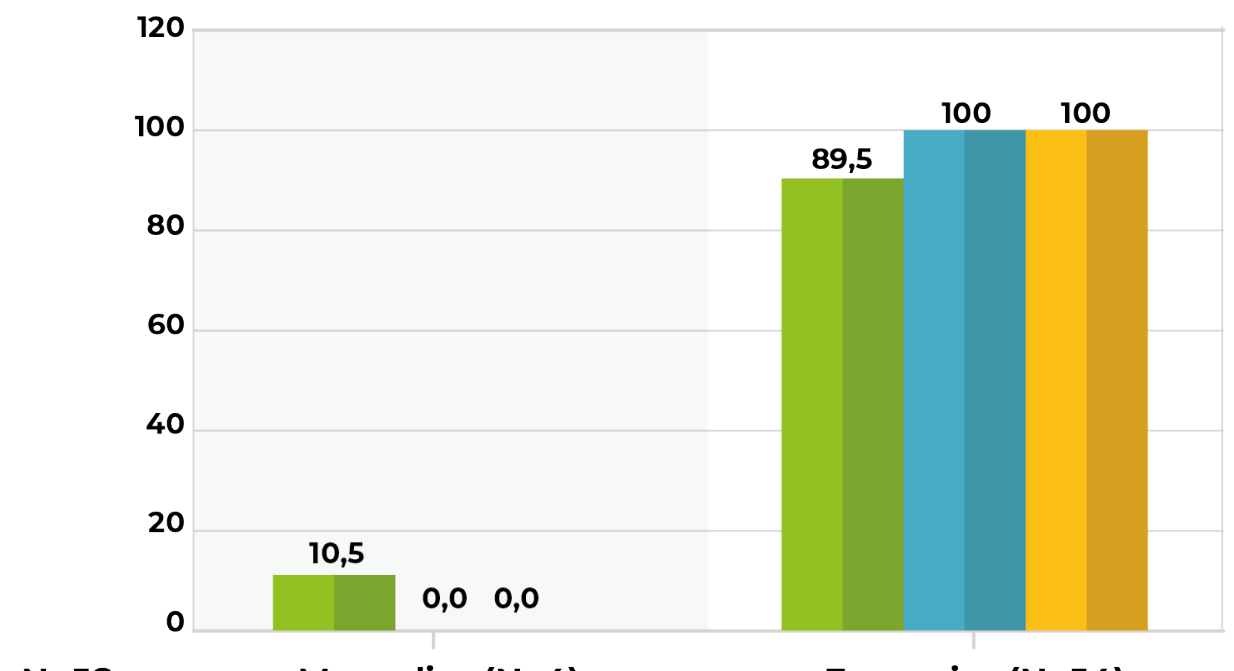

\section{$\mathbf{N}=\mathbf{5 8}$}

Masculino( $\mathbf{N}=4)$

Femenino( $N=54)$

Normocalcemia $(n=38)$

Hipoparatiroidismo Transitorio( $n=8)$

Hipoparatiroidismo Permanente(n=12)

Gráfico 8: Distribución de sexo por diagnostico a los 6 meses.

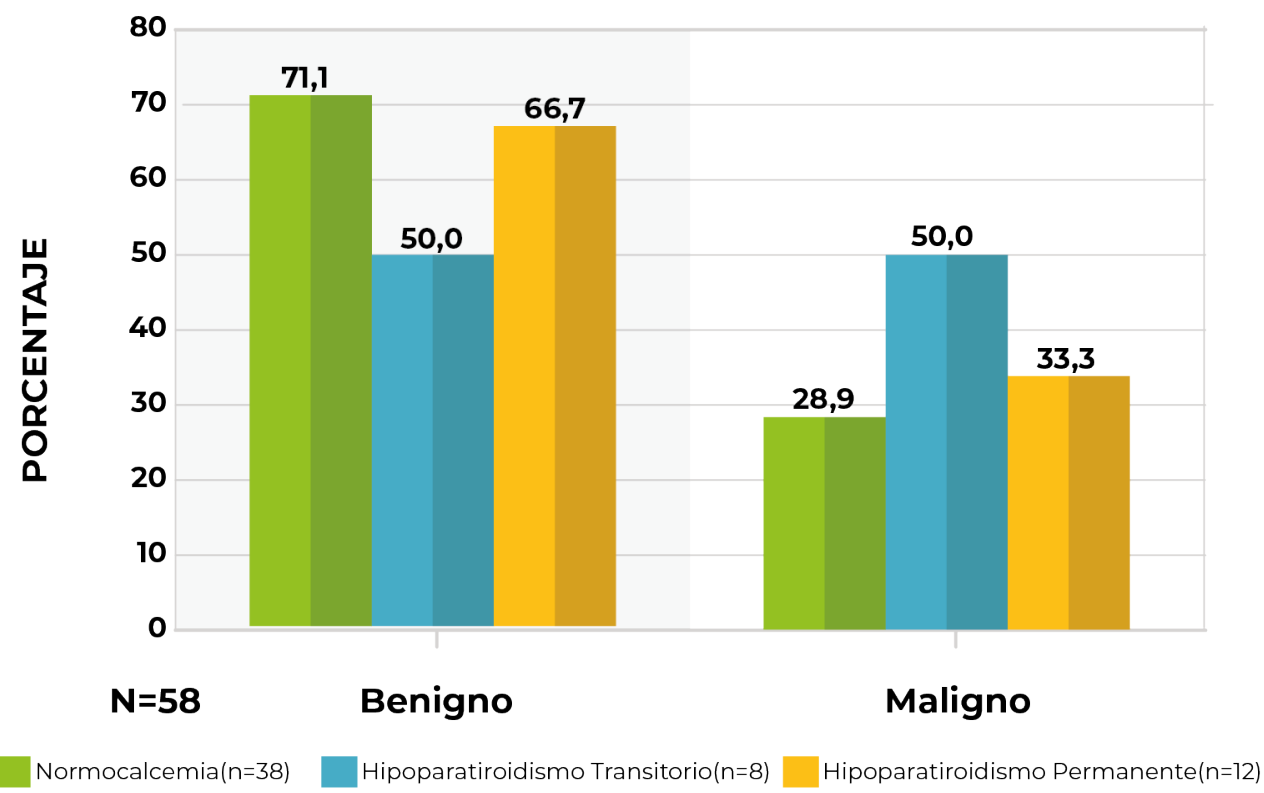

Gráfico 9: Distribución de características histológicas por diagnóstico a los 6 meses.

En los pacientes con HP según Anatomía Patológica el 41,7\% fueron clasificados como "Bocio Coloide Multinodular" y el $63,2 \%$ de los pacientes con NC también presentaron el mismos diagnóstico según anatomía patología, mientras que los pacientes con HT según Anatomía Patológica el 50\% tuvo un resultado de "Carcinoma Papilar de Tiroides" (Gráfico 10).

Según PAAF el 28,9\% presentó de los 
pacientes $\mathrm{NC}$ presentaron diagnóstico de "Atipia de significado indeterminado"; los pacientes con HT el 50\% presentaron un diagnóstico de insatisfactorio o no contaba con el estudio, mientras que en los pacientes con HP, el 33,3\% fueron clasificados como "Benignas" (Gráfico 11).

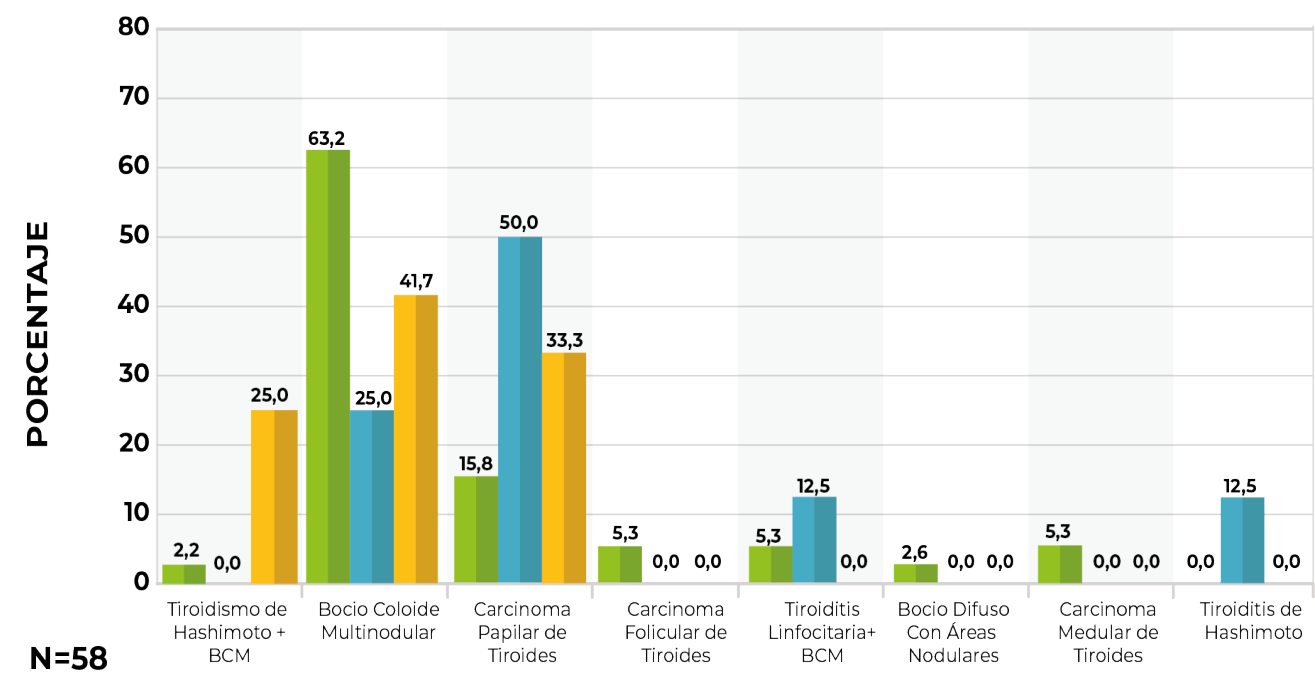

Normocalcemia(n=38) Hipoparatiroidismo Transitorio(n=8) Hipoparatiroidismo Permanente $(n=12)$

Gráfico 10: Distribución de características de Anatomía Patológica por diagnóstico a los 6 meses.

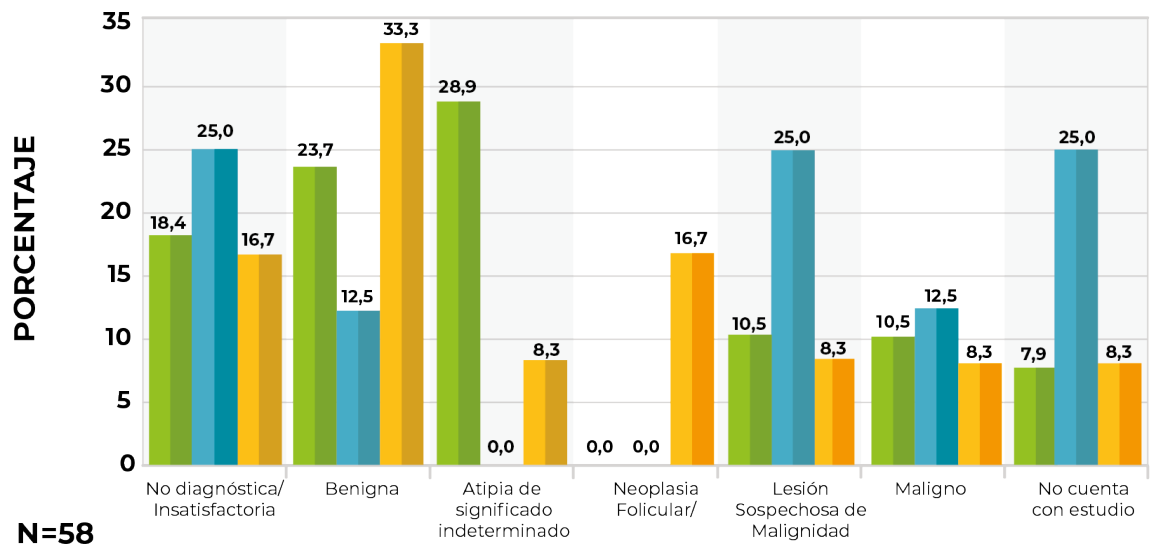

Normocalcemia(n=38) Hipoparatiroidismo Transitorio $(n=8) \quad$ Hipoparatiroidismo Permanente $(n=12)$

Gráfico 11: Distribución de características de PAAF_BETHESDA por diagnóstico a los 6 meses.

Se encontró que los pacientes con hipocalcemia evaluados por calcio total a las 24 hs presentan 16 veces más de riesgo de presentar Hipoparatiroidismo permanente. Mientras que pacientes con hipocalcemia evaluados por calcio iónico a las $24 \mathrm{hs}$ y 96hs presentan 90,2 y 45,22 veces más de riesgo de presentar Hipoparatiroidismo permanente, respectivamente (Tabla 2) 
Tabla 2: Razón de probabilidades de la hipocalcemia antes de los 6 meses con respecto al Hipoparatiroidismo permanente.

\begin{tabular}{|c|c|c|c|c|c|c|c|c|}
\hline \multicolumn{2}{|c|}{ Parámetro de riesgo } & \multicolumn{2}{|c|}{$\begin{array}{c}\text { Hipoparatiroidismo } \\
\text { Permanente } \\
(\mathrm{n}=12 ; \mathbf{2 0}, \mathbf{7 \%})\end{array}$} & \multicolumn{2}{|c|}{$\begin{array}{c}\text { Hipoparatiroidismo } \\
\text { No Permanente } \\
(n=46 ; 79,3 \%)\end{array}$} & \multicolumn{2}{|c|}{$\begin{array}{c}\text { Total }(n=58 ; \\
100 \%)\end{array}$} & \multirow[t]{2}{*}{ OR } \\
\hline Dosaje & Interpretación & $\mathbf{n}$ & $\%$ & $\mathbf{n}$ & $\%$ & $\mathbf{n}$ & $\%$ & \\
\hline \multirow{2}{*}{$\begin{array}{l}\text { Calcio total } \\
24 \mathrm{~h}\end{array}$} & Hipocalcemia & 9 & 56,3 & 7 & 43,8 & 16 & 27,6 & \multirow{2}{*}{16,71} \\
\hline & Normocalcemia & 3 & 7,1 & 39 & 92,9 & 42 & 72,4 & \\
\hline \multirow{2}{*}{$\begin{array}{l}\text { Calcio total } \\
48 \mathrm{~h}\end{array}$} & Hipocalcemia & 12 & 60,0 & 8 & 40,0 & 20 & 34,5 & \multirow{2}{*}{$\mathrm{NC}$} \\
\hline & Normocalcemia & 0 & 0,0 & 38 & 100,0 & 38 & 65,5 & \\
\hline \multirow{2}{*}{$\begin{array}{l}\text { Calcio total } \\
96 \mathrm{~h}\end{array}$} & Hipocalcemia & 12 & 66,7 & 6 & 33,3 & 18 & 31,0 & \multirow{2}{*}{$\mathrm{NC}$} \\
\hline & Normocalcemia & 0 & 0,0 & 40 & 100,0 & 40 & 69,0 & \\
\hline \multirow{2}{*}{$\begin{array}{l}\text { Calcio iónico } \\
24 \mathrm{~h}\end{array}$} & Hipocalcemia & 11 & 68,8 & 5 & 31,3 & 18 & 27,6 & \multirow{2}{*}{90,2} \\
\hline & Normocalcemia & 1 & 2,4 & 41 & 97,6 & 40 & 72,4 & \\
\hline \multirow{2}{*}{$\begin{array}{c}\text { Calcio iónico } \\
\quad 48 \mathrm{~h}\end{array}$} & Hipocalcemia & 12 & 60,0 & 8 & 40,0 & 20 & 34,5 & \multirow{2}{*}{ NC } \\
\hline & Normocalcemia & 0 & 0,0 & 38 & 100,0 & 38 & 65,5 & \\
\hline \multirow{2}{*}{$\begin{array}{c}\text { Calcio iónico } \\
96 \mathrm{~h}\end{array}$} & Hipocalcemia & 11 & 55,0 & 9 & 45,0 & 20 & 34,5 & \multirow{2}{*}{45,22} \\
\hline & Normocalcemia & 1 & 2,6 & 37 & 97,4 & 38 & 65,5 & \\
\hline
\end{tabular}

En cuanto a la correlación en función del CAT, se puedo observar que esta aumenta a medida que la evaluación se realiza de forma más tardía. La medición de calcio total a las $24 \mathrm{hs}, 48 \mathrm{hs}$ presentaron una correlación moderada para predecir Hipoparatiroidismo permanente evaluado por PTH a los 6 meses $(r=0,532$ y $r=0,668$, respectivamente), sin embargo a las $96 \mathrm{hs}$ esta correlación aumenta a alta $(\mathrm{r}=0,734)$. Por el contrario, la medición de CAI como marcador de correlación disminuye en función a si el control, ya que se encontró una correlación alta de predecir el Hipoparatiroidismo permanente medido por PTH a los 6 meses a las $24 \mathrm{hs}$ alta $(\mathrm{r}=0,719)$ y disminuye a moderado al evaluar calcio iónico medido a las $48 \mathrm{hs}$ y $96 \mathrm{hs}(\mathrm{r}=0,663$ y $r=0,578$, respectivamente). En todos los casos la correlación fue significativa $(p<0,05)$.

Tabla 3: Correlación entre el dosaje de calcio total e iónico con el diagnóstico de Hipoparatiroidismo permanente medido por el dosaje de hormona paratiroidea a los 6 meses.

\begin{tabular}{|c|c|c|c|c|c|c|c|c|c|}
\hline \multicolumn{2}{|c|}{ Parámetro de riesgo } & \multicolumn{2}{|c|}{$\begin{array}{c}\text { Hipoparatiroidismo } \\
\text { Permanente } \\
(\mathrm{n}=12 ; 20,7 \%)\end{array}$} & \multicolumn{2}{|c|}{$\begin{array}{c}\text { Hipoparatiroidismo } \\
\text { No Permanente } \\
(\mathrm{n}=46 ; \mathbf{7 9 , 3 \% )}\end{array}$} & \multicolumn{2}{|c|}{$\begin{array}{c}\text { Total }(n=58 \\
100 \%)\end{array}$} & \multirow[t]{2}{*}{$r$} & \multirow[t]{2}{*}{$p$} \\
\hline Dosaje & Interpretación & n & $\%$ & n & $\%$ & $\mathbf{n}$ & $\%$ & & \\
\hline \multirow{2}{*}{$\begin{array}{l}\text { Calcio total } \\
24 \mathrm{~h}\end{array}$} & Hipocalcemia & 9 & 56,3 & 7 & 43,8 & 16 & 27,6 & \multirow[b]{2}{*}{0,532} & \multirow[b]{2}{*}{0,00} \\
\hline & Normocalcemia & 3 & 7,1 & 39 & 92,9 & 42 & 72,4 & & \\
\hline \multirow{2}{*}{$\begin{array}{c}\text { Calcio total } \\
\quad 48 \mathrm{~h}\end{array}$} & Hipocalcemia & 12 & 60,0 & 8 & 40,0 & 20 & 34,5 & \multirow{2}{*}{0,668} & \multirow{2}{*}{0,00} \\
\hline & Normocalcemia & 0 & 0,0 & 38 & 100,0 & 38 & 65,5 & & \\
\hline \multirow{2}{*}{$\begin{array}{l}\text { Calcio total } \\
\quad 96 \mathrm{~h}\end{array}$} & Hipocalcemia & 12 & 66,7 & 6 & 33,3 & 18 & 31,0 & \multirow{2}{*}{0,734} & \multirow{2}{*}{0,001} \\
\hline & Normocalcemia & 0 & 0,0 & 40 & 100,0 & 40 & 69,0 & & \\
\hline \multirow{2}{*}{$\begin{array}{c}\text { Calcio iónico } \\
24 \mathrm{~h}\end{array}$} & Hipocalcemia & 11 & 68,8 & 5 & 31,3 & 18 & 27,6 & \multirow{2}{*}{0,719} & \multirow[t]{2}{*}{0,00} \\
\hline & Normocalcemia & 1 & 2,4 & 41 & 97,6 & 40 & 72,4 & & \\
\hline
\end{tabular}




\begin{tabular}{ccrrrrrrrr} 
Calcio iónico & Hipocalcemia & 12 & 60,0 & 8 & 40,0 & 20 & 34,5 & 0,663 & 0,001 \\
$48 \mathrm{~h}$ & Normocalcemia & 0 & 0,0 & 38 & 100,0 & 38 & 65,5 & & \\
Calcio iónico & Hipocalcemia & 11 & 55,0 & 9 & 45,0 & 20 & 34,5 & 0,578 & 0,001 \\
$96 \mathrm{~h}$ & Normocalcemia & 1 & 2,6 & 37 & 97,4 & 38 & 65,5 & & \\
\hline
\end{tabular}

Correlación (r): Correlación Kappa

\section{DISCUSIÓN}

La hipocalcemia posoperatoria es la complicación más frecuente después de la tiroidectomía total 19 al $38 \%$, en la mayoría de los casos transitoria y limitada a las primeras semanas del procedimiento quirúrgico. La incidencia de hipoparatiroidismo permanente varia, según las distintas casuísticas, del 0 al 3\% $(1,11,19)$. En este estudio, el primero en reportar la incidencia de hipocalcemia e hipoparatiroidismo en el Instituto de Previsión Social Hospital Central, se encuentra que la incidencia de hipoparatiroidismo transitorio fue del $13,8 \%$ y de un $20,7 \%$ para el hipoparatiroidismo permanente, afectando a esta ultima la calidad de vida del paciente, determinando una prolongada estancia hospitalaria y la necesidad de terapia suplementaria con calcio y/o vitamina D durante el resto de la vida. (12-14). Existen muchos estudios realizados que tienen como objetivo identificar factores clínicos, anatomopatológicos y bioquímicos en grado de predecir el desarrollo de hipoparatiroidismo permanente. $(10,15)$. En la literatura médica se ha referido que la histología maligna de la lesión tiroidea, el hipertiroidismo, la extirpación accidental de la paratiroides, la re intervención y/o la disección de cuello están directamente relacionados con el desarrollo de hipoparatiroidismo permanente tras tiroidectomía. $(15,16)$. En nuestra experiencia no se han encontrado asociaciones estadísticamente significativas entre el desarrollo de hipoparatiroidismo permanente y los factores anteriormente citados. Sin embargo, el establecimiento de estos factores predictores continúa siendo un desafío.

La mayor incidencia de morbilidad entre los pacientes con diagnostico citológico de malignidad es consistente con estudios previos. (15) En nuestro estudio se observó que la incidencia de citología maligna fue del 33,3\% similar al diagnóstico de citología benigna de 33,3\%, no hubo diferencia importante alguna para desarrollo de hipoparatiroidismo. Algunos autores creen que, ante la presencia de malignidad, se tiende a ser más exhaustivo en la resección de tejido tiroideo durante la cirugía, lo cual conduce a una mayor probabilidad de paratiroidectomía incidental o de isquemia de las paratiroides que a su vez, con lleva a hipoparatiroidismo. (10). La hipocalcemia fue significativamente mayor para los pacientes de sexo femenino, a pesar que la proporción de género en nuestro estudio fue predominantemente mujeres (17) frente a los hombres (10) $89,5 \%$ versus el 10,5\%. La asociación entre hipoparatiroidismo y sexo femenino se puede deber a que las mujeres son más propensas a presentar patologías tiroideas y además presentar déficit de calcio y vitamina D por enfermedades asociadas como menopausia y osteoporosis que conllevan a déficit de dichos elementos. (18).

El valor de paratohormona (PTH) y valores bajos plasmáticos de calcemia y fosfatemia en el posoperatorio también han sido relacionados, según algunos estudios, 
con el desarrollo de hipoparatiroidismo permanente. $(10,15)$ De nuestra investigación emerge que una calcemia en las 24 horas de la intervención quirúrgica medida con calcio iónico por debajo del límite normal es un factor altamente pronóstico de hipoparatiroidismo permanente, como ya se han referido en otras casuísticas. (10, 11). Se determinó respecto a la hipocalcemia medido con calcio total que un descenso a las 24 horas presento un valor predictivo de hipoparatiroidismo permanente pero más aún un descenso a las 96 horas,

\section{CONCLUSIONES}

La mayor parte de los pacientes eran mujeres, el promedio de edad fue de $52 \pm 14$ años, las tres cuartas partes son del área urbana. 6 de cada 10 pacientes eran hipertensos. La mediana de tiempo de internación fue de 3 días.

En cuanto a la función de la glándula, los indicadores evaluados presentaron valores de normalidad según THS, T3 T4. Según histología, la mayoría de los pacientes presentan una característica benigna, alrededor de la mitad presentó Bocio Coloide Multinodular, según Anatomía patológica mientras que según Ecografía, la mayoría presentó Bocio multinodular. Una mayor parte presenta diagnóstico de normocalcemia a los 6 meses, mientras pero dichos descensos no fueron muy significativos. $(19,20)$. Al contrario, en la experiencia de algunos autores la evolución de la calcemia determinada en los primeros días después de la intervención no parece dar suficientes informaciones sobre el posible desarrollo de hipoparatiroidismo permanente, mientras que en muchos artículos se ha enfatizado sobre el valor pronóstico de la determinación de PTH, no obstante, el elevado coste y la complejidad del método de laboratorio. $(11,15)$

que el porcentaje presentó hipoparatiroidismo transitorio fue menor que el de hipoparatiroidismos permanentes. Se puedo observar que todos los pacientes presentan dosaje normal de calcio total e ionizado preoperatorio. En cuanto a las características de los pacientes, se resalta que solo las mujeres presentaron hipoparatiroidismo permanente. La probabilidad de presentar Hipoparatiroidismo permanente es mayor en pacientes con hipocalcemia medido por calcio iónico a las $24 \mathrm{hs}$ y $96 \mathrm{hs}$ presentan y calcio total a las $24 \mathrm{hs}$.

La medición de calcio total de calcio iónico medido presentó una correlación moderada para predecir Hipoparatiroidismo permanente, evaluado por PTH a los 6meses.

\section{REFERENCIAS}

1. Marcucci G, Cianferotti L, Brandi ML. Clinical presentation and management of hypoparathyroidism. Best Practice \& Research Clinical Endocrinology \& Metabolism. 2018; in press.

\section{Espiarda S, Vantyghema}

MC, Desailloud R. Actualisation sur l'hypoparathyroïdie : un peu de théorie, beaucoup de pratique. Annales

\section{d'Endocrinologie $78 \quad$ (2017) $\quad$ S1-S10}

3. Sikjaer T, Moser E, Rolighed L, Underbjerg L, Sofie L, Mosekilde L, Rejnmark L. Concurrent Hypoparathyroidism Is Associated With Impaired Physical Function and Quality of Life in Hypothyroidism. Journal of Bone and Mineral Research. 2016: 1-9

4. Htun HM, Edmiston R; Kaimal K, 
Kumar N. Variable management of postoperative hypoparathyroidism at nine NHS trusts in north west England: the need for a universal protocol. Otorrinolaringología clínica. doi: 10.1111 / coa.13205

5. Selberherr A; Niederle B. Vermeidung und Management des Hypoparathyreoidismus nach Schilddrüsenoperationen. Chirurg. 2014; 1:4

6. Cusano N, Bilezikian J. Sign and Symptoms of hypoparathroidism. Endocrinol Metab Clin N Am 47 (2018) 759-770

7. Barquero-Melchor $\mathrm{H}$, Delgado-Rodríguez M, Juantá-Castro J. Hipocalcemia e hipoparatiroidismo post-tiroidectomía. Acta Médica Costarricense. Acta méd costarric. 2015; 57 (4): 184-89

8. Shoback DM, Bilezikian JP, Costa AG, Dempster D, Dralle H, Khan AA, Bouillon, R et al. Presentación del hipoparatiroidismo: etiologías y características clínicas. The Journal of Clinical Endocrinology \& Metabolism. 2016; 101 (6): 2300-2312. doi: 10.1210 / jc.2015-3909

9. Hadiza S, Ann J. Surgical Hypoparathyroidism. Endocrinol Metab Clin N Am. 2018; 2:14

10. Nam Cho J, Seo W, Young S. Predictors and risk factors of hypoparathyroidism after total thyroidectomy. International Journal of Surgery. 2016; 34: 47-52

11. Manoela K, Luongo L, Roberto C, Garcia L, Furtado V. Postoperative calcium levels as a diagnostic measure for hypoparathyroidism after total thyroidectomy. Arch Endocrinol Metab. 2015;59/5: 428:433

12. Langner $E$, Tincani $A$, del Negro $A$. Use of prophylactic oral calcium after total thyroidectomy: a prospective study. Arch Endocrinol Metab. 2017;61/5: 447-454
13. Leinung $M$, Beyer T. Postoperative Hypocalcemia After Thyroidectomy: Can It Be Prevented?. Commentary Endocr Pract. 2015; 21 (4) 452-3

14. Clarke B. Epidemioogy and complications of hipoparathyroidism. Endocrinol Metab Clin N Am. 2018; 1:12

15. Eismontas V, Slepavicius A, Janusonis V, Zeromskas P, Beisa V, Strupas K, et al. Predictors of postoperative hypocalcemia occurring after a total thyroidectomy: results of prospective multicenter study. Eismontas et al. BMC Surgery. 2018; 18 (55): 2-12 https://doi. org/10.1186/s12893-018-0387-2

16. Mannstadt $\mathrm{M}$, Bilezikian J, Thakker R, Hannan F, Clarke B, Reijnmark L, et al. Hypoparathyroidism. NATURE REVIEWS. 2017; 3 (17): 1:20

17. Coimbra C, Monteiro F, Oliverira P, Ribeiro L, Giesteira M, Conde A. Hypoparathyroidism following thyroidectomy: Predictive factors. Acta Otorrinolaringol Esp. 2016. http://dx.doi.org/10.1016/j.otorri.2016.06.008

18. Orloff L, Wiseman S, Bernet V, Fahey T, Shaha A, Shindo M, et al. American Thyroid Association Statement on Postoperative Hypoparathyroidism: Diagnosis, Prevention, and Management in Adults. Tiroides. 2018; 28 (7), 830-841. doi: 10.1089 / thy.2017.0309

19. Clarke B, Brown E, Collins M, Jüppner H, Lakatos P, Romanischen A, Thakker R. Epidemiology and Diagnosis of Hypoparathyroidism. J Clin Endocrinol Metab. 2016: 7-9

20. Gafni R, Collins M. Hypoparathyroidism. N Engl J Med 2019;380:1738 